\title{
An Extension to the Sliding Short Method of Connector and Adaptor Evaluation*
}

\author{
Glenn F. Engen* \\ Institute for Basic Standards, National Bureau of Standards, Boulder, Colorado 80302
}

(October 18, 1971)

\begin{abstract}
Sliding short methods represent a measurement tool of substantial potential for the measurement of small losses such as are associated with waveguide connectors or adaptors. Until recently, however, the use of these methods has been inhibited by the uncertainty of the error contribution due to nonideal short behavior.

A recent analysis by Almassy has shown that by the use of proper techniques, the error contribution from this source is usually negligible, provided that the adaptor (or connector) is "well matched."

It is the purpose of this paper to eliminate this latter restriction, develop additional measurement methods, and describe further applications.
\end{abstract}

Key words: Adaptor; connector; efficiency; sliding short.

\section{Introduction}

A measurement problem, of continuing interest in the microwave art, is that of adaptor evaluation. With the advent of the power equation $[1]^{1}$ methods and use of terminal invariant parameters [2], the dependence upon impedance properties is suppressed, and the dissipative characteristic emerges as the parameter of major interest. A similar observation may be made with regard to connectors. Here the dissipation at the connector interface, or more specifically, the lack of loss repeatability, represents a basic limitation to the attainable accuracy in many microwave measurements.

The application of sliding short methods, to this measurement problem, has been known for some time $[3,4]$. These techniques were generalized in conjunction with the development of the power equation methods [1, 2]. Despite this revival of interest, however, the technique has been subject to a major limitation: an ideal sliding short is assumed.

In practice, of course, no such device exists. Moreover, the losses in the short may be of the same order (or larger) than those in the joint or adaptor to be evaluated. In the absence of further information, this loss represents a potential source of substantial error.

A recent analysis by Almassy [5] has shown that, with proper techniques, the sliding short losses can

${ }^{*}$ Senior Research Scientist, Electromagnetics Division, National Bureau of Standards, Boulder, Colo. 80302.

${ }^{1}$ Figures in brackets indicate the literature references at the end of this paper. be substantially reduced, provided that the adaptor (or joint) reflection is small (i.e., $\left|S_{11}\right| \ll 1,\left|S_{22}\right| \ll 1$ ).

It is the purpose of this paper to eliminate this restriction, develop additional measurement methods, and describe further applications.

\section{Background}

The major thrust of this paper will be directed towards the measurement of the efficiency of an arbitrary two-port. In particular this two-port may be a connector, waveguide-coax adaptor, etc. By detinition, the efficiency is the ratio of the (net) power output, to the (net) power input, and is a function of the load (but not of the source) impedance. Throughout this paper, the efficiency is assumed to be large (small losses). (In general the accuracy of this method decreases rapidly with losses greater than $10 \mathrm{~dB}$.)

It will prove convenient to briefly review the existing theory. The recommended instrumentation takes the form shown in figure 1 . The basic configuration will

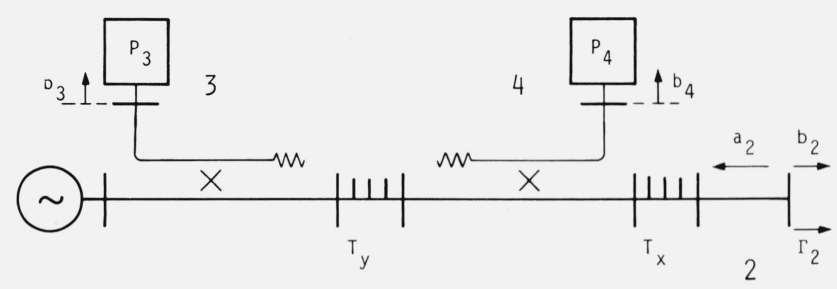

FigURE 1. Generalized reflectometer for use in efficiency measurement. 
be recognized as a generalized reflectometer (g-reflectometer), where the junction parameters are arbitrary except as subsequently noted. In particular, the tuning transformers $T_{x}, T_{y}$ permit one to impose certain conditions on the measurement system. The device to be evaluated is connected to port 2 , while arms 3 and 4 are terminated by power meters.

Although power meters have been specified for arms 3 and 4 , it will prove convenient, momentarily, to assume an alternative detection scheme such that the complex ratio $b_{3} / b_{4}$ is measured, where $b_{3}, b_{4}$ are the emergent wave amplitudes. Let arm 2 be terminated by a moving short $\left(\Gamma_{2}=e^{j \theta}\right)$, and let the values $\left(\frac{b_{3}}{b_{4}}\right)$ be plotted in the complex plane as $\theta$ is permitted to vary. The resultant locus is a circle, an example of which is shown in figure 2 . The parameters of this locus, in particular the radius of the circle, $R$, and the (absolute) distance, $R_{c}$, between its center and the origin, play a major role in the efficiency measurement.

Inspection of figure 2 indicates that ${ }^{2}$

$$
\begin{aligned}
& \left|\frac{b_{3}}{b_{4}}\right|_{\max }=R+R_{c}, \\
& \left|\frac{b_{3}}{b_{4}}\right|_{\min }=R-R_{c} .
\end{aligned}
$$

and solving for $R, R_{c}$ yields:

$$
\begin{aligned}
& R=\frac{1}{2}\left(\left|\frac{b_{3}}{b_{4}}\right|_{\text {max }}+\left|\frac{b_{3}}{b_{4}}\right|_{\text {min }}\right), \\
& R_{c}=\frac{1}{2}\left(\left|\frac{b_{3}}{b_{4}}\right|_{\max }-\left|\frac{b_{3}}{b_{4}}\right|_{\text {min }}\right) .
\end{aligned}
$$

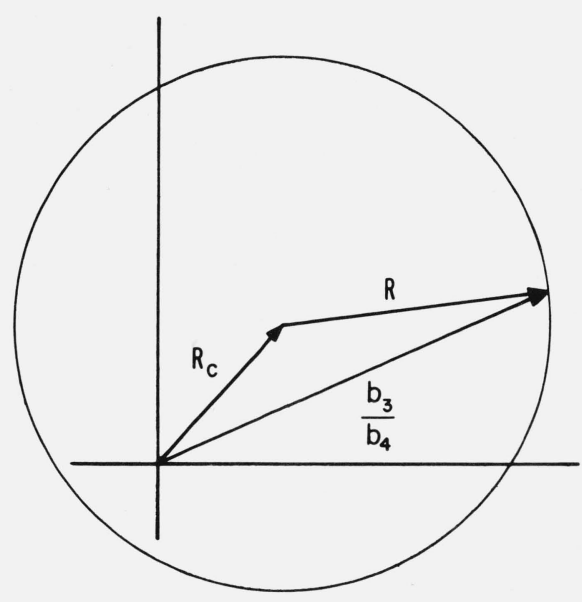

Figure 2. Locus of $\mathrm{b}_{3} / \mathrm{b}_{4}$ as a function of $\theta$.
The determination of $R, R_{c}$ thus involves amplitudes only, the phase detection capability is not required. Finally, the power meters respond to the square of the amplitude such that $P_{3}=\left|b_{3}\right|^{2}$ etc.

In order to make an efficiency measurement, the two-port is connected as shown in figure 3. Note that the designations have been chosen such that port 2 of the four arm junction mates with port 2 of the twoport. The "load" impedance, for which the efficiency is measured, is actually provided by the four arm junction.

To be more specific, it is convenient to postulate that $P_{4}$ is constant. (In practice this is often done by a "leveling" or feedback arrangement.) The source impedance, $\Gamma_{g}$, for the "equivalent" generator, which now obtains at port 2, depends upon $T_{x}$ and the adjacent coupler [6], but is independent of $T_{y}$ and the remaining coupler. The efficiency, which is obtained in the measurement described in the following paragraph, is that for an assumed power flow from terminal 1 to terminal 2 , with the termination $\Gamma_{g}$. It will be denoted by $\eta_{21}\left(\Gamma_{g}\right)$.

The efficiency measurement calls for connecting a moving short to terminal 1 and determining the radius, $R_{1}$, of the resulting circular locus using (3). The twoport is then removed, and the operation repeated at terminal 2 to obtain $R_{2}$. It has been shown [2] that

$$
\eta_{21}\left(\Gamma_{g}\right)=R_{1} / R_{2}
$$

In an alternative method, it is convenient (but not essential) to use the configuration shown in figure 4 . Here the efficiency, $\eta_{12}\left(\Gamma_{l}\right)$, is measured for a power flow from terminal 2 to terminal 1 , and for the terminating load $\Gamma_{l}$.

The measurement procedure now calls for the adjustment of $T_{x}$ such that $P_{3}$ vanishes. The parameters, $R, R_{c}$, of the circular locus are then measured with the moving short connected to terminals 1 and 2 . It will be shown (see appendix) that $\eta_{12}\left(\Gamma_{l}\right)$ is given by:

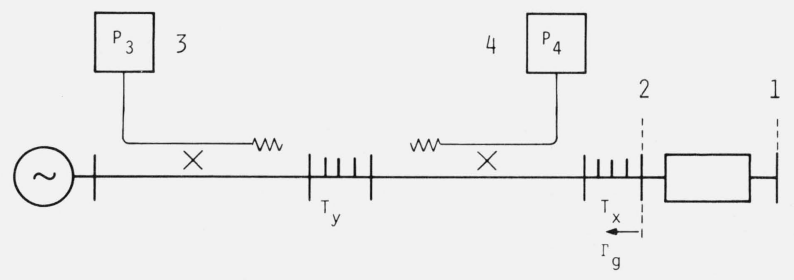

FIGURE 3. G-reflectometer with two-port connected.

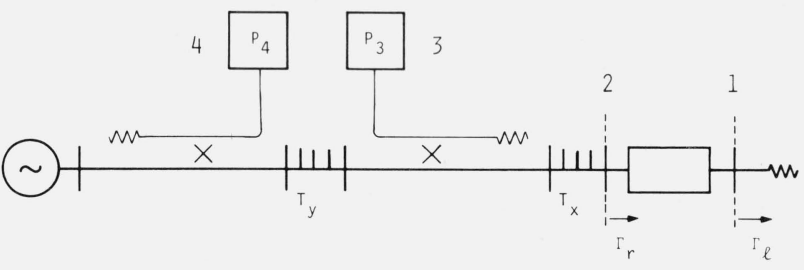

FigURE 4. Alternative configuration for g-reflectometer. 
In order to account for non-ideal behavior, the model here assumed is:

$$
\Gamma=r e^{j \psi} e^{-2(\alpha+j \beta) l}
$$

where $r, \psi$ are the magnitude and phase of the plunger reflection, $\alpha, \beta$ are the loss and phase constants of the line in which the "short" moves, and $l$ is its position with respect to the reference flange.

If (8) is substituted in (7), the resulting relation can be written as follows:

$$
\begin{aligned}
\frac{b_{3}}{b_{4}}= & \frac{b-a c^{*} r^{2} e^{-4 \alpha l}}{1-|c|^{2} r^{2} e^{-4 \alpha l}} \\
& +\frac{(a-b c) r e^{j \psi} e^{-2(\alpha+j \beta) l}\left(1+c^{*} r e^{-j \psi} e^{-2(\alpha-j \beta) l}\right)}{\left[1-|c|^{2} r^{2} e^{-4 \alpha l}\right]\left(1+c r e^{j \psi} e^{-2(\alpha+j \beta) l}\right)}
\end{aligned}
$$

where $(*)$ denotes the complex conjugate.

Although this expression is a complicated one, a great deal can be learned about the problem by inspection of this result. First, if the short is ideal, $r=1, \psi=\pi, \alpha=0$, and the expression becomes:

$$
\frac{b_{3}}{b_{4}}=\frac{b-a c^{*}}{1-|c|^{2}}-\frac{(a-b c) e^{-2 j \beta l}\left(1-c^{*} \mathrm{e}^{2 j \beta l}\right)}{\left(1-|c|^{2}\right)\left(1-c e^{-2 j \beta l}\right)} .
$$

Here it is noted that $b_{3} / b_{4}$ is the sum of two terms. The first is a constant (as $l$ varies), while the second is of constant magnitude but variable phase. (Note that the last factors in the numerator and denominator of the second term are conjugates of each other.) This explicitly demonstrates the previously stated result: the locus of $b_{3} / b_{4}$ is a circle of radius

$$
R=\left|\frac{a-b c}{1-|c|^{2}}\right|
$$

and distance to the center

$$
R_{c}=\left|\frac{b-a c^{*}}{1-|c|^{2}}\right|
$$

Next, it is desirable to return to the more general relationship contained in (9) and to consider the functional dependence of $\left|b_{3} / b_{4}\right|$ upon $l$. It is this relationship which is plotted in figure 6 .

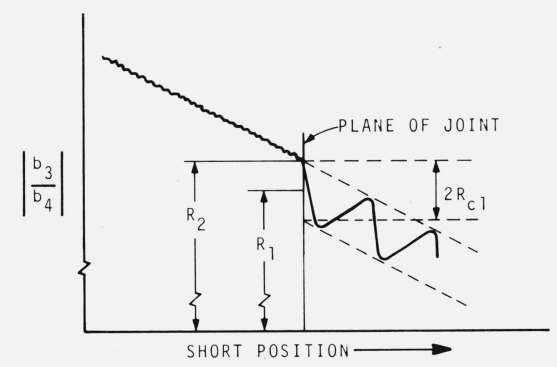

FigURE 6. "Typical" response in an actual measurement.
In the discussion which follows, it will prove useful to consider not only the functional relationship of (9), but also the family of functions which is obtained from (9) for different values of $\psi$. Conceptually, one assumes the measurement is repeated a number of times, where the moving "shorts" have the same value for $r$, but differing values of $\psi$.

For a given $l$, the value of $\left|b_{3} / b_{4}\right|$ will depend upon $\psi$, and for some particular choice of $\psi$ will have the maximum value:

$$
\left|\frac{b_{3}}{b_{4}}\right|_{\max }=\frac{|a-b c| r e^{-2 \alpha l}+\left|b-a c^{*} r^{2} e^{-4 \alpha l}\right|}{1-|c|^{2} r^{2} e^{-4 \alpha l}} .
$$

For some other choice of $\psi,\left|b_{3} / b_{4}\right|$ will have the minimum ${ }^{4}$ :

$$
\left|\frac{b_{3}}{b_{4}}\right|_{\min }=\frac{|a-b c| r e^{-2 \alpha l}-\left|b-a c^{*} r^{2} e^{-4 \alpha l}\right|}{1-|c|^{2} r^{2} e^{-4 \alpha l}} .
$$

For each value of $l$, there will be some member of the family (value of $\psi$ ) for which these maximum and minimum values are realized, but these limits are never exceeded. Equations (13), (14) are evidently the envelope of the family. If this envelope is denoted by $E$, then:

$$
E=\frac{|a-b c| r e^{-2 \alpha l} \pm\left|b-a c^{*} \mathrm{r}^{2} e^{-4 \alpha l}\right|}{1-|c|^{2} r^{2} e^{-4 \alpha l}} .
$$

Because of the oscillatory behavior of $\left|b_{3} / b_{4}\right|$, the envelope may be easily inferred from a single member of the family provided that several cycles, or more, have been recorded. From (15), the extrapolation of this envelope to the plane where $l=0$ yields:

$$
\left.E\right|_{l=0}=\frac{|a-b c| r \pm\left|b-a c^{*} r^{2}\right|}{1-|c|^{2} r^{2}} .
$$

The use of this result to obtain approximate values for the efficiency will be evaluated in the following section. It is next instructive to consider the general behavior of $E$ as $l$ varies.

Returning to (15), the first term in the numerator tends to dominate the entire expression, and provides a simple exponential decay. The second term in the numerator determines the amplitude associated with the oscillatory behavior. If $c$ vanishes, this amplitude is constant, while if $b$ vanishes, the amplitude decays exponentially. If both these conditions obtain the oscillatory behavior is absent. The most distinctive situation occurs when $b$ and $a c^{*}$ are nominally equal. In this case there will be some value of $l$ (which may or may not be physically realizable) for which this second term will be a minimum (possibly zero).

Finally, the second term in the denominator is usu-

${ }_{4}$ This assumes that the first term exceeds the second which, for the applications envisioned, is usually the case. 
ally small ${ }^{5}$ with respect to unity. As a whole the denominator increases as $l$ increases, and the value of the complete expression decreases somewhat more rapidly than would otherwise be the case. As a rule, this effect is rather small. The most general form of the envelope is a distorted hyperbola as shown in figure 7.

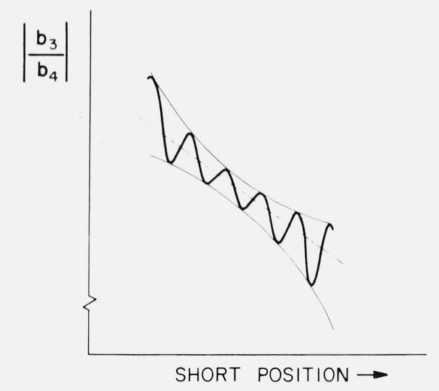

FigURE 7. General form of system response.

\section{Practical Considerations}

As already noted, the measurement technique calls for recording $\left|b_{3} / b_{4}\right|$ as a function of $l$ (short displacement). The envelope of this curve is then projected to the plane where $l=0$, and $E_{\max }, E_{\min }$ substituted for $\left|b_{3} / b_{4}\right|$ in (3), (4). The resulting approximations to $R, R_{c}$ are denoted by primes and given by:

$$
\begin{gathered}
R^{\prime}=\frac{|a-b c|(1-\epsilon)}{1-|c|^{2}(1-\epsilon)^{2}} \\
R_{c}^{\prime}=\frac{\left|b-a c^{*}(1-\epsilon)^{2}\right|}{1-|c|^{2}(1-\epsilon)^{2}},
\end{gathered}
$$

where $r=1-\epsilon$, and $\epsilon$ is usually a small quantity.

The evaluation of the error, $e$, due to nonzero $\epsilon$, in an efficiency determination using (5), begins with the definition

$$
e=\frac{\frac{R_{1}^{\prime}}{R_{2}^{\prime}}-\frac{R_{1}}{R_{2}}}{\frac{R_{1}}{R_{2}}} .
$$

Substitution of (11), (17) into this result leads to

$$
e \simeq 2 \epsilon\left(\left|c_{2}\right|^{2}-\left|c_{1}\right|^{2}\right)
$$

where the subscripts 1,2 , differentiate between the $g$-reflectometer parameters which obtain at ports 1 and 2 respectively, and only the first order terms have been retained.

If $\left|c_{1}\right|,\left|c_{2}\right|, \epsilon$ were known, their values could be substituted into (20) and $e$ would become a "correction factor" rather than error. As a rule, however, the

5 It is not within the scope of this paper to consider the many "pathological" situations which could be invented, e.g., couplers without directivity or reversed in direction, extreme departures from impedance match conditions, etc. Because the mathematical formulation is general enough to include these cases, these additional qualifying statements are required.

potential accuracy improvement does not warrant the extra effort this requires. As a general guideline, in the configuration of figure $3,|c| \simeq\left|\Gamma_{u}\right|$ where $\Gamma_{u}$ is a reflection coefficient which is "representative" of the two-port (e.g., adaptor) and the load for which the efficiency is measured. In the configuration of figure $4,|c|$ is determined by the adjustment of $T_{y}$; if this is such as to minimize the amplitude of the oscillatory behavior of $\left|b_{3} / b_{4}\right|$ in response to the short motion, the foregoing guideline $\left(|c| \simeq\left|\Gamma_{u}\right|\right)$ is again satisfied. ${ }^{6}$

For an adaptor of VSWR of 1.5, a typical value of $|c|$ is 0.2 , while $\epsilon$ will ordinarily be less than 0.01 . The maximum expected error, due to the nonideal short is thus 0.1 percent over a wide range of practical operating conditions, and the typical error is probably 0.01 percent or less.

To complete this discussion, there will also be an error in the determination of $R_{c 1}, R_{c 2}$ due to the nonideal short. Ordinarily the error contribution from this source will be one or more magnitudes smaller than that already described and thus negligible.

Thus far, the discussion has implicitly assumed that the same short is used at both terminals 1 and 2 . In some applications, the evaluation of a waveguidecoax adaptor for example, this is obviously impossible. In this case, the generalization of $(20)$ is

$$
e \simeq\left(\epsilon_{2}-\epsilon_{1}\right)+2\left(\epsilon_{2}\left|c_{2}\right|^{2}-\epsilon_{1}\left|c_{1}\right|^{2}\right) .
$$

Here $\epsilon_{1}, \epsilon_{2}$ are associated with the shorts used at ports 1 and 2 respectively.

It will be immediately recognized that the difference between $\epsilon_{1}$ and $\epsilon_{2}$ is now a potential source of substantial error. Fortunately, however, this error can be eliminated. The procedure is to make the measurement by each of the two described procedures (using (5) and (6)). ${ }^{7}$ Assuming that the same pair of shorts are used for each of these measurements, it is easily shown that the geometric mean of the two efficiency determinations gives the desired result. Conversely, the square root of the ratio of these measurements gives the ratio between the reflection coefficient magnitudes of the respective plungers.

Returning to the expression for efficiency, it is convenient to write (5) in the form:

$$
\eta_{21}=1-\frac{R_{2}-R_{1}}{R_{2}}
$$

This shows that the vertical displacement between $R_{2}$ and $R_{1}$ (fig. 6) is a direct measure of the difference between $\eta_{21}$ and unity. Typically, this difference is no more than a few percent, and the performance require-

\footnotetext{
${ }^{6}$ Although this guideline should prove adequate for most practical purposes, an "exact" evaluation of $c_{1}, c_{2}$ is not difficult to obtain. In particular, the generator in figures 3 or 4 is replaced by a variable termination which is adjusted such that $b_{4}$ vanishes when the system is excited via port 1 (or 2). The reflection coefficient observed at port 1 (or 2) is now $-c_{1}$ (or $\left.-c_{2}\right)$.

${ }^{7}$ This assumes that in the first of the described methods $T_{x}$ has been adjusted such that " $\Gamma_{g}$ " equals the load reflection coefficient for which $\eta_{21}$ is desired. Moreover, it will be recognized that, during the second measurement, the two ports of the adaptor are reversed, recognized that, during the second measurement, the two ports of the adaptor are reversed,
in relation to the g-reflectometer, as compared with their positions during the first
} 
ments on the associated measurement system are primarily for resolution and stability rather than "absolute" accuracy. For example, a $10 \%$ error in either $R_{2}$ or in $\left(R_{2}-R_{1}\right)$ will cause an error of only a few tenths of a percent in $\eta_{21}$. Conversely, a system resolution of a part in $10^{4}$ is required if $\left(R_{2}-R_{1}\right)$ is to be observed with a resolution of one percent, etc.

The role of tuner $T_{y}$ has been referred to a number of times. Although the operation is, in theory, independent of this adjustment, certain second order error considerations have been noted. In addition, however, is the practical requirement of matching the excursions of $\left|b_{3} / b_{4}\right|$ to the dynamic range limitations of the $x-y$ recorder. If these excursions are permitted to be large relative to $R_{2}-R_{1}$, the resolution, with which $R_{2}-R_{1}$ is measured, suffers. With a little experience this adjustment is not a difficult task.

Finally, it is of interest to note that the recording provides not only an indication of the joint or other losses, but also an indication of the loss of the transmission line in which the short moves. In many cases, this provides a useful confirmation of the system sensitivity and calibration. Indeed, it is possible to measure the loss in terms of the waveguide attenuation/wavelength without otherwise calibrating the system.

\section{Applications}

This technique represents a powerful tool for certain measurement problems. An immediate example, already alluded to, is the evaluation of connector loss. In order to gain some insight into the behavior of the Type $\mathrm{N}$ connector, a coaxial line was devised with a continuous outer conductor and a slip joint on the inner conductor such as is found in the Type $\mathrm{N}$ connector. This joint showed a loss of $0.006 \mathrm{~dB}$ at $9 \mathrm{GHz}$. In another test piece, the center conductor was continuous, while the outer conductor included a replica of the joint found in the Type N. This exhibited a loss of $0.05 \mathrm{~dB} .{ }^{8}$ The measured loss of a complete "Type N" joint, in which the slots had been deleted from the outer sleeve, was $0.01 \mathrm{~dB}$.

This technique has also found application in the evaluation of certain parameters associated with a cryogenic noise source developed for the Comsat Corporation. In particular the corrected value of the temperature at the output port required both the loss per unit length in the output waveguide, and the loss in a waveguide window. Both of these quantities were easily measured via these methods.

Another use of these methods is in conjunction with the large antenna gain calibration project at the Jet Propulsion Laboratory [6]. Here a loss correction factor is obtained for the 30-40 foot waveguide run between the gain standard horn and the reference plane. The mismatch corrections are also explicitly accounted for with this technique.

${ }^{8}$ Although the purpose of these measurements was ostensibly that of evaluating the con nector design, the real motivation was that of demonstrating the flexibility of the measure ment technique. The above result is rather surprising, and may not be actually representativ of the general design. In any case, it appears desirable to further pursue this subject.

\section{Summary}

The usefulness of the sliding short methods of "attenuation" measurement has been substantially enhanced by application of the first order correction theory, for nonideal short behavior, developed by Almassy [5], and the subsequent extension outlined in this paper.

The microwave art is rapidly approaching the place, if indeed it is not already there, where connector imperfections represent a major barrier to further advances in the accuracy of microwave measurements. Although a great deal of recent effort has been expended in the direction of improved VSWR specifications, the more important parameter, for many applications at least, is the dissipation characteristic. This method should prove a particularly valuable tool in assessing the magnitude of this problem.

The procedure may be implemented in a variety of ways, the major requirement is for a high degree of stability in the measuring system. One such system has been described in some detail by Almassy ${ }^{9}$ [5]. At the Jet Propulsion Laboratory, a commercial version of the "precision insertion loss test set" developed at JPL [7] has been adapted for this use.

The existence of this first order correction theory also makes it possible to envision the extension of sliding short methods to much lower frequencies than has previously been feasible. In particular, because the theory explicitly takes account of line loss, it may prove useful to construct the inner and outer conductors in the form of a helix. Another possibility is that of immersing the line in a liquid dielectric.

Finally, the tuning adjustments, called out in this procedure, can be eliminated if a phase detection capability, such as is found in network analyzers, is assumed. Much of the detail for doing this has been worked out, but remains to be implemented.

\section{Appendix}

The purpose of this appendix is to derive (6).

Referring to figure 4 , the relationship between $b_{3} / b_{4}$ and the reflection coefficient, $\Gamma_{r}$, presented at terminal 2 by the two-port and its load, $\Gamma_{l}$, may be written, ${ }^{10}$

$$
\frac{b_{3}}{b_{4}}=\frac{A \Gamma_{r}+B}{C \Gamma_{r}+D}
$$

where $A, B, C, D$ are parameters of the $g$-reflectometer. In a similar way, the relationship between $\Gamma_{r}$ and $\Gamma_{l}$ is given by,

$$
\Gamma_{r}=\frac{a \Gamma_{l}+b}{c \Gamma_{l}+1}
$$

\footnotetext{
${ }^{9}$ The "compensation" included in Almassy's system has not been found necessary in these measurements.

10 The numerator and denominator could obviously be divided by $D$ to obtain better agreement with the functional form of (7). The existing form, however, provides better continuity with earlier work in this area.
} 
where for the moment, $\Gamma_{l}$ is assumed to be arbitrary, and $a, b, c$ are parameters of the two-port whose efficiency is required. (Note that this represents a change in terminology from (7).)

The required efficiency is given by [2],

$$
\eta_{12}\left(\Gamma_{l}\right)=\frac{|a-b c|\left(1-\left|\Gamma_{l}\right|^{2}\right)}{\left|1+c \Gamma_{l}\right|^{2}-\left|a \Gamma_{l}+b\right|^{2}} .
$$

By use of (11), (12), and a little algebra, it can be shown that

$$
R_{2}\left(1-\left(\frac{R_{c 2}}{R_{2}}\right)^{2}\right)=\frac{|A|^{2}-|B|^{2}}{|A D-B C|} .
$$

To obtain the counterpart expression involving $R_{1}, R_{c 1}$ it is convenient to substitute (24) into (23) which (for an arbitrary $\Gamma_{l}$ ) yields,

$$
\frac{b_{3}}{b_{4}}=\frac{(A a+B c) \Gamma_{l}+(A b+B)}{(C a+D c) \Gamma_{l}+(C b+D)} .
$$

This is in the same form as (23) so,

$R_{1}\left(1-\left(\frac{R_{c 1}}{R_{1}}\right)^{2}\right)$

$=\frac{|A a+B c|^{2}-|A b+B|^{2}}{|(A a+B c)(C b+D)-(A b+B)(C a+D c)|} \cdot$

By hypothesis, however, $b_{3}$ vanishes when $\Gamma_{l}$ represents the load reflection for which the efficiency is desired. Therefore,

$$
(A b+B)=-(A a+B c) \Gamma_{l},
$$

from which,

$$
\frac{B}{A}=-\frac{a \Gamma_{l}+b}{c \Gamma_{l}+1} .
$$

Substitution of this result into (28), (26) leads to,

$R_{1}\left(1-\left(\frac{R_{c 1}}{R_{1}}\right)^{2}\right)$

$$
=\frac{|A| \cdot|a-b c| \cdot\left(1-\left|\Gamma_{l}\right|^{2}\right)}{\left|1+c \Gamma_{l}\right| \cdot\left|C\left(a \Gamma_{l}+b\right)+D\left(1+c \Gamma_{l}\right)\right|},
$$

$R_{2}\left(1-\left(\frac{R_{c 2}}{R_{2}}\right)^{2}\right)$

$$
=\frac{|A| \cdot\left(\left|1+c \Gamma_{l}\right|^{2}-\left|a \Gamma_{l}+b\right|^{2}\right)}{\left|1+c \Gamma_{l}\right| \cdot\left|C\left(a \Gamma_{l}+b\right)+D\left(1+c \Gamma_{l}\right)\right|} \cdot
$$

Finally, comparison of the ratio of (31) to (32) with (25) yields (6).

\section{References}

[1] Engen, G. F., Power equations: A new concept in the description and evaluation of microwave systems, IEEE Trans. Instr. Meas. IM-20, 49-57 (Feb. 1971).

[2] Engen, G. F., An introduction to the description and evaluation of microwave systems using terminal invariant parameters, Nat. Bur. Stand. (U.S.), Monogr. 112, 26 pages (Oct. 1969).

[3] Cullen, A. L., Measurement of microwave transmission efficiency, Wireless Engineer 26, 255-258 (Aug. 1949).

[4] Beatty, R. W., Determination of attenuation from impedance measurements, Proc. IRE, 38, 895-897 (Aug. 1950).

[5] Almassy, G., A first order correction to sliding short behavior with application to the problem of measuring small losses, IEEE Trans. Instr. Meas. IM-20, 162-169 (Aug. 1971).

[6] Batelaan, P. D., private communication.

[7] Stelzried, C. T., Reid, M. S., and Petty, S. M., A precision dc potentiometer microwave insertion loss test set, IEEE Trans. Instr. Meas. IM- 15, 98-104 (Sept. 1966).

(Paper 75C3\&4-324) 